\title{
Síndrome de Ativação Macrofágica em Pacientes com Artrite Idiopática Juvenil(*)
}

\section{Macrophage Activation Syndrome in Patients with Juvenile Idiopathic Arthritis}

\author{
Rogério do Prado ${ }^{(1)}$, Maria Teresa R. A. Terreri ${ }^{(2)}$, Cláudio A. Len ${ }^{(2)}$, Josefina Braga ${ }^{(3)}$ e Maria Odete E. Hilário ${ }^{(4)}$
}

\section{RESUMO}

A síndrome de ativação macrofágica (SAM) é uma complicação rara das doenças reumáticas crônicas, particularmente a artrite idiopática juvenil (AIJ) de início sistêmico. Este processo pode ser desencadeado por agentes infecciosos virais e bacterianos, neoplásicos, drogas antiinflamatórias não esteroidais ou drogas modificadoras da doença, mudanças abruptas das medicações e doenças reumáticas. O quadro clínico inicia-se com irritação do sistema nervoso central, acompanhado de falências hepática e renal, além de pancitopenia. Relatamos três casos de pacientes com AIJ do nosso serviço que desenvolveram SAM com descrição das características clínicas, evolutivas e de tratamento.

Palavras-chave: síndrome de ativação macrofágica, artrite idiopática juvenil, hemofagocitose, ciclosporina A, crianças.

\section{INTRODUÇÃO}

A síndrome de ativação macrofágica (SAM) ou síndrome hemofagocítica reativa é uma complicação aguda e rara das doenças reumáticas crônicas, particularmente da artrite idiopática juvenil (AIJ) de início sistêmico, podendo estar associada à significativa mortalidade ${ }^{(1,2)}$.

Uma série de pacientes com proliferação histiocitária benigna, distinta da histiocitose maligna, foi primeiramente descrita por Risdall, et al., em 1979, que atribuíram esta condição a uma desregulação imunológica induzida por uma infecção viral em hospedeiro imunocomprometido ${ }^{(3)}$.

\begin{abstract}
The macrophage activation syndrome (MAS) is an uncommon complication of chronic rheumatic diseases, specially systemic onset juvenile idiopathic arthritis (JIA). It can be triggered by infectious (viral or bacterial) or malignant diseases, non-steroidal anti-inflammatory or disease modified anti-rheumatic drugs, changes in the therapy and rheumatic diseases. The clinical features present at the onset are related mainly with central nervous system involvement, hepatic and renal failure and pancytopenia. We describe the clinical, evolutive features and treatment of three patients with JIA that developed MAS.
\end{abstract}

Keywords: macrophage activation syndrome, juvenile idiopathic arthritis, hemophagocytosis, children, cyclosporine.

O termo SAM foi descrito pela primeira vez por Hadchouel, et al, em 1985, em um relato de sete pacientes com AIJ de início sistêmico que desenvolveram esta complicação no decorrer da doença ${ }^{(4)}$.

A SAM é um distúrbio do sistema fagocitário mononuclear caracterizado por ativação e excessiva proliferação dos linfócitos $\mathrm{T}$ e macrófagos, levando ao aumento da produção de citocinas, responsáveis pelas principais manifestações clínicas ${ }^{(2,5)}$. Em 1997 foi descrito no Japão um caso de SAM associado com AIJ de início sistêmico com grande produção de Fator de Necrose Tumoral $-\alpha$ levando à ativação macrofágica ${ }^{(6)}$. Além disso, o fator

\footnotetext{
Setor de Reumatologia Pediátrica da disciplina de Alergia, Imunologia Clínica e Reumatologia da Universidade Federal de São Paulo/Escola Paulista de Medicina (Unifesp/EPM). Recebido em 24/06/2003. Aprovado, após revisão, em 04/03/2004.

1. Médico pós-graduando da Unifesp/EPM.

2. Professor assistente da Unifesp/EPM.

3. Professor adjunto de especialidade pediátrica e responsável pelo setor de Hematologia Pediátrica da Unifesp/EPM.

4. Professora livre docente da Unifesp/EPM.
} 
estimulador de colônias de macrófagos está elevado na fase ativa da doença e diminuído na fase convalescente, indicando que este fator possa ser um potente estimulador de produção de citocinas pelos macrófagos ${ }^{(6,7)}$.

Embora a etiologia da SAM seja desconhecida, sabe-se que este processo pode ser desencadeado por agentes infecciosos virais (Epstein-Barr vírus, parvovírus B19, varicela zóster, citomegalovírus) e bacterianos, neoplásicos, drogas antiinflamatórias não esteroidais (ácido acetilsalicílico) ou drogas modificadoras de doença [sais de ouro, sulfassalazina ou metotrexate (MTX) ${ }^{(8)}$, mudanças abruptas das medicações e doenças reumáticas. Em alguns casos pode ocorrer espontaneamente ${ }^{(1,2,5,9)}$.

As manifestações clínicas são dramáticas e iniciam-se com irritação do sistema nervoso central. As alterações neurológicas são caracterizadas por irritabilidade, comprometimento progressivo do sensório, até coma e alteração da personalidade. Ocorre um rápido desenvolvimento de falência hepática, sangramentos, púrpuras e equimoses. Falência renal, com presença de hematúria e proteinúria poderá ocorrer. Alguns dos sintomas lembram o quadro da AIJ sistêmica como febre, hepatoesplenomegalia e adenomegalia.

O diagnóstico diferencial da SAM inclui condições com características clínico-laboratoriais semelhantes como AIJ, vasculites, infecções virais (citomegalovírus, vírus da hepatite $\mathrm{B}$, da imunodeficiência humana, herpes, varicela e adenovírus), bacterianas (salmonela, ricketsia e enterobactérias), parasitoses (leishmania e plasmódio) e fungos (aspergillus, cândida e histoplasma $)^{(5)}$.

Laboratorialmente há presença de anemia, trombocitopenia, leucopenia, queda da velocidade de hemossedimentação (VHS), elevação de enzimas hepáticas e evidência de distúrbios de coagulação como o aumento dos tempos de protrombina e tromboplastina parcial, níveis elevados de produtos de degradação da fibrina, hipofibrinogenemia e deficiência dos fatores de coagulação. O diagnóstico é feito pelo mielograma, cuja característica patognomônica é a presença de numerosos macrófagos bem diferenciados fagocitando elementos hematopoiéticos na medula óssea ${ }^{(9)}$. Ocorre também a presença de infiltrado de linfócitos e fagócitos em vários tecidos como os gânglios e em órgãos vitais como sistema nervoso central e figado.

Em virtude da gravidade desta síndrome, o seu reconhecimento deve ser imediato e o tratamento deve ser iniciado o mais precocemente, pois o risco de uma fatalidade é alto. Não há um protocolo para o tratamento da SAM em pacientes com doenças sistêmicas. Há um consenso que a instabilidade hidroeletrolítica e desordens de coagulação (hipofibrinogenemia e trombocitopenia) ${ }^{(10)}$ têm de ser corrigidas rapidamente e o paciente deve ser assistido em unidade de tratamento intensivo. As infecções, quando possível, devem ser tratadas. A imunoglobulina polivalente tem sido descrita como sendo efetiva em alguns trabalhos ${ }^{(2,3)}$. Os antiinflamatórios não hormonais devem ser descontinuados, assim como o MTX. Alguns autores têm preconizado o uso de corticosteróides, na forma de pulsoterapia com metilprednisolona $30 \mathrm{mg} / \mathrm{kg} / \mathrm{dose}$ por 3 dias, e mais recentemente o uso da ciclosporina $\mathrm{A}^{(2,8,9,11)}$. Prahalad, et al. descreveram um paciente com SAM e AIJ de início sistêmico tratado com anti-fator de necrose tumoral $-\alpha$ (etanercept). O seguimento deste paciente sugere que o etanercept pode ser um agente terapêutico efetivo no tratamento da $\operatorname{SAM}^{(7)}$.

Em uma série de 24 crianças com diagnóstico de SAM, 18 apresentaram AIJ sistêmica. Todos os pacientes tiveram febre como manifestação inicial, 14/24 apresentaram hepatoesplenomegalia e 12/24 anormalidades neurológicas (coma e cefaléia). Pulsoterapia com metilprednisolona induziu à remissão em $15 / 21$ pacientes. A ciclosporina $A$ foi efetiva e usada nos pacientes em que o tratamento com corticosteróide endovenoso falhou, com boa resposta. Dois pacientes faleceram ${ }^{(2)}$.

Outros autores também descreveram um total de quatro crianças com AIJ que desenvolveram SAM com as mesmas características clínico-laboratoriais ${ }^{(5,7,8,11)}$.

Em virtude da raridade de casos descritos na literatura, apresentamos três pacientes do nosso serviço com descrição das características clínicas, evolutivas e de tratamento.

\section{RELATO DO CASO 1}

DMB, sexo feminino, branca, 16 anos, natural e procedente de São Paulo iniciou AIJ poliarticular com quatro anos de idade. Inicialmente apresentou artrite em tornozelo esquerdo (pós-trauma) e duas semanas após evoluiu com artrite em tornozelo direito e punhos, acompanhada de febre. Usou prednisona (2,5 $\mathrm{mg}$ em dias alternados), naproxeno e cloroquina. Posteriormente apresentou artrite em pequenas articulações, cotovelos, ombros, coluna cervical, joelhos e quadris. Nesse período foi introduzido MTX. Desde então, vinha evoluindo com períodos de melhora e piora, com fisioterapia irregular e grande dificuldade de aderência ao tratamento por razões familiares. Exames da época do diagnóstico apresentavam hemoglobina: 9,1g/dLdL; hematócrito: 29\%; leucócitos: $6.600 / \mu 1$ 
com distribuição normal; plaquetas: $280.000 / \mathrm{mm}^{3}$; VHS: $58 \mathrm{~mm}$; fator anti-núcleo e fator reumatóide: negativos; transaminase glutâmico-oxalacética (TGO): 12 U/1 (normal até $38 \mathrm{U} / 1$ ); transaminase glutâmico-pirúvica (TGP): $7 \mathrm{U} / 1$ (normal até $41 \mathrm{U} / 1$ ); proteínas totais: $6,2 \mathrm{~g} / \mathrm{dL}$ (normal: 6,4 a $8,1 \mathrm{~g} / \mathrm{dL}$ ); albumina: 2,6 g/dL (normal: 3,2 a 5,6 $\mathrm{g} / \mathrm{dL}$ ); alfa 2 globulina: $0,19 \mathrm{~g} / \mathrm{dL}$ (normal: 0,58 a 0,76 $\mathrm{g} / \mathrm{dL}$ ); gama-globulina:1,86 g/dL (normal: 0,7 a 1,6 g/dL); urina I: normal. Após três anos foi substituído o MTX pela ciclosporina A e houve importante melhora clínica e laboratorial. Entretanto, após dois anos parou a medicação por conta própria e o quadro voltou a piorar, sendo reintroduzido MTX (até $1 \mathrm{mg} / \mathrm{kg} / \mathrm{sem}$, via oral) associado a sulfassalazina $(20 \mathrm{mg} / \mathrm{kg} / \mathrm{dia})$ com melhora gradativa. Após três anos, com o esquema acima, posteriormente a um quadro de infecção das vias aéreas superiores, desenvolveu SAM, manifestada por anemia, sangramento gengival importante, dispnéia e dor torácica. O mielograma apresentava hiperplasia da série eritrocitária e megacariocítica. Foi hospitalizada e tratada com pulsoterapia de metilprednisolona (30 $\mathrm{mg} / \mathrm{kg} / \mathrm{dose}$ ) por três dias e ciclosporina A endovenosa. Exames da época mostravam: hemoglobina: $5,4 \mathrm{~g} / \mathrm{dL}$; hematócrito: $19 \%$; leucócitos: $2.100 / \mu \mathrm{l}$ (1\% bastonetes; $32 \%$ segmentados; $1 \%$ eosinófilos; $61 \%$ linfócitos; $1 \%$ monócitos; $4 \%$ basófilos); plaquetas: $5.000 / \mathrm{mm}^{3}$; reticulócitos: 5 ; VHS: $9 \mathrm{~mm}$; TGO: $54 \mathrm{U} / \mathrm{l}$; TGP $47 \mathrm{U} / \mathrm{l}$; creatinina $0,7 \mathrm{mg} / \mathrm{dL}$. Evoluiu bem e atualmente está em uso de leflunomide há dois anos com melhora clínica e laboratorial. Exames atuais: hemoglobina: 11,6g/dL; hematócrito: 35\%; leucócitos: 5.180/ $\mu$ l (diferencial normal); plaquetas: $186.000 / \mathrm{mm}^{3}$; VHS: $25 \mathrm{~mm}$; TGO: $23 \mathrm{U} / \mathrm{l}$; TGP: $7 \mathrm{U} / \mathrm{l}$; gama glutamiltransferase: $13 \mathrm{U} / 1$ (normal até $42 \mathrm{U} / 1$ ).

\section{RELATO DO CASO 2}

RASL, sexo feminino, branca, 9 anos e 8 meses, natural e procedente de São Paulo. Com 3 anos de idade iniciou AIJ sistêmica (febre $39-40^{\circ} \mathrm{C}$, rash cutâneo, hepatoesplenomegalia e linfoadenomegalia, artrite em punhos, joelhos e tornozelos). Foram realizados exames que mostraram hemoglobina: 8,1g/dL; hematócrito: 32\%; leucócitos: $14.300 / \mu 1$ com distribuição normal; plaquetas: 167.000/ $\mathrm{mm}^{3}$; VHS: $110 \mathrm{~mm}$; fator anti-núcleo: negativo; fator reumatóide: negativo; desidrogenase lática: $361 \mathrm{U} / 1$ (normal até $460 \mathrm{U} / 1$ ); proteínas totais: $5,4 \mathrm{~g} / \mathrm{dL}$; albumina: $2,72 \mathrm{~g} / \mathrm{dL}$; alfa-2 globulina: $0,92 \mathrm{~g} / \mathrm{dL}$; gama-globulina: $1,32 \mathrm{~g} / \mathrm{dL}$; Urina I: normal. Foi realizada pulsoterapia com metilprednisolona $30 \mathrm{mg} / \mathrm{kg} /$ dose por três dias, iniciado indometacina $3 \mathrm{mg} / \mathrm{kg} /$ dia, prednisona $20 \mathrm{mg} /$ dia (1 mg/ $\mathrm{kg} / \mathrm{dia}$ ) e MTX $5 \mathrm{mg} / \mathrm{sem}$. Como a paciente evoluiu com melhora clínica e laboratorial iniciou-se redução gradativa do corticosteróide oral com suspensão após seis meses. Após um mês da suspensão do corticosteróide pela melhora clínica, foi suspensa indometacina. Após três meses voltou a apresentar febre diária $38,5-39^{\circ} \mathrm{C}$, sendo reintroduzida indometacina e corticosteróide $10 \mathrm{mg}$ /dia e mantido MTX. Após uma semana com febre apresentou queda do estado geral, dor abdominal e cefaléia occipital de forte intensidade acompanhada de vômitos, confusão mental e sinais meníngeos, tendo sido internada. Os exames mostraram hemoglobina: 8,5 g/dL; hematócrito: 27\%; leucócitos: $16.700 / \mu 1$ (diferencial normal); plaquetas: $175.000 / \mathrm{mm}^{3}$; VHS: $5 \mathrm{~mm}$; TGO: 56 U/1; TGP: 45 U/1; Urina I: normal, líquor normal. Realizado mielograma que apresentava medula rica em células com hiperplasia eritroblástica com agrupamentos de eritroblastos, além de normoplasia granular e megacariocítica e hipoplasia linfocítica e estabelecido diagnóstico de SAM. Foi indicada pulsoterapia com metilprednisolona $30 \mathrm{mg} / \mathrm{kg} /$ dose, ciclosporina A $3 \mathrm{mg} / \mathrm{kg} /$ dia endovenosa, aumentada dose de corticosteróide oral $(1 \mathrm{mg} / \mathrm{kg} / \mathrm{dia})$ e suspenso MTX. Apresentou melhora do quadro, evoluindo bem. Após um mês os exames laboratoriais mostravam: hemoglobina: 10,1 g/dL; hematócrito: 33\%; leucócitos:

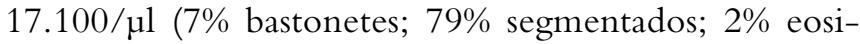
nófilos; $10 \%$ linfócitos; $2 \%$ basófilos); plaquetas: $304.000 /$ $\mathrm{mm}^{3}$; VHS: $66 \mathrm{~mm}$; urina I: normal, tendo sido iniciada redução do corticosteróide.

\section{RELATO DO CASO 3}

DC, sexo masculino, branco, 15 anos e 3 meses, natural e procedente de São Paulo, com 5 anos iniciou AIJ sistêmica apresentando febre acima de $39^{\circ} \mathrm{C}$, rash cutâneo, hepatoesplenomegalia, artrite em joelhos, tornozelos, cotovelos e punhos. Os exames iniciais mostraram hemoglobina: 6,6

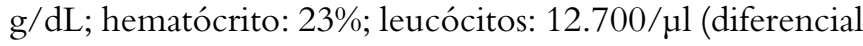
normal); plaquetas: $635.000 / \mathrm{mm}^{3}$; VHS: $89 \mathrm{~mm}$; fator antinúcleo: negativo; fator reumatóide: negativo; anti-DNA: negativo; urina I: normal. Foi iniciado naproxeno $19 \mathrm{mg} /$ $\mathrm{kg} /$ dia e deflazacort $9 \mathrm{mg} /$ dia. Foi introduzido MTX até $0,75 \mathrm{mg} / \mathrm{kg} / \mathrm{sem}$ subcutâneo (SC) em virtude da artrite persistente em joelhos, tornozelos e interfalangeanas proximais. Em razão da má aderência, o paciente retorna após dez meses com artrite em joelhos e tornozelo direito. Foi introduzida pulsoterapia com metilprednisolona $30 \mathrm{mg} /$ $\mathrm{kg} /$ dose por três dias e mantido o restante da medicação. 
Após sete meses, pela piora clínica (artrite em punho direito, joelhos e tornozelos) e laboratorial (anemia, aumento das provas inflamatórias) foi introduzido leflunomide $100 \mathrm{mg} /$ dia por três dias e manutenção com 20 mg/dia. Após um mês em virtude do quadro de febre, rash e anemia foi internado. Realizados dois mielogramas, sendo o primeiro normal e o segundo (após 25 dias) apresentando discreta hipoplasia eritroblástica com maturação preservada, normoplasia linfoplasmocitária, hipoplasia megacariocítica, com plaquetogênese preservada. Foram realizados exames que mostraram hemoglobina: $9.2 \mathrm{~g} / \mathrm{dL}$; hematócrito: $28 \%$; leucócitos: 3000/ $\mu \mathrm{l}$ (7\% bastonetes; $73 \%$ segmentados; $18 \%$ linfócitos; $2 \%$ basófilos); plaquetas: $52.000 / \mathrm{mm}^{3}$; VHS: $1 \mathrm{~mm}$; TGO: 813U/1; TGP $252 \mathrm{U} / 1$. Foi feita a hipótese de SAM e suspensa a medicação anterior. Realizadas quatro pulsoterapias de metilprednisolona $(30 \mathrm{mg} / \mathrm{kg} / \mathrm{dose})$, ciclosporina A $3,5 \mathrm{mg} / \mathrm{kg} /$ dia endovenosa e prednisolona 0,83 $\mathrm{mg} / \mathrm{kg} /$ dia endovenosa com boa evolução.

\section{DISCUSSÃO}

A síndrome de ativação macrofágica é uma complicação rara e pouco conhecida da AIJ. Descrevemos três casos de crianças com SAM diagnosticada clínica e laboratorialmente.

Embora as infecções sejam os fatores desencadeantes mais referidos ${ }^{(2,3,10,11)}$, apenas o caso 1 apresentou quadro de IVAS precedendo a sintomatologia. Outro fator descrito, são as medicações como ocorreu no caso 3, que após a introdução de uma nova droga (leflunomide) iniciou o quadro clínico. Entretanto, não sabemos se isto foi uma coincidência ou se a SAM pode ter sido desencadeada pelo MTX ou eventualmente uma infecção concomitante. Dados de literatura mostram que nem sempre são encontrados fatores desencadeantes para o início do quadro clínico e laboratorial ${ }^{(2,5)}$. Ravelli, et al. descreveram um paciente com AIJ de início sistêmico que desenvolveu SAM rapidamente após a introdução de MTX, sugerindo que esta droga possa ser um potencial desencadeador da síndrome ${ }^{(8)}$. Stéphan, et al. descreveram como desencadeador freqüente a infecção em $11 / 24$ pacientes e a introdução de medicação em três $\operatorname{casos}^{(2)}$.

Dois pacientes nossos (casos 2 e 3) apresentaram febre e queda do estado geral mimetizando o quadro de AIJ sistêmica. No nosso estudo apenas um paciente apresentou alteração neurológica. Na literatura a freqüência de alterações neurológicas variam de letargia até coma, em 50\% dos pacientes ${ }^{(2)}$. Em outros relatos de caso não foram descritas essas manifestações ${ }^{(4,8,10,11)}$.
Com relação às alterações laboratoriais, a anemia ocorreu em todos os pacientes, a plaquetopenia em $2 / 3$ pacientes $\mathrm{e}$ a leucopenia em apenas um, mostrando uma característica importante da síndrome que é a destruição de células sangüíneas por macrófagos na medula óssea. A pancitopenia é um dos fatores essenciais para o diagnóstico diferencial com a atividade da forma sistêmica da AIJ. Outra alteração laboratorial é a queda importante da VHS que ocorreu em todos os pacientes, e que também é um dado contra a possibilidade de atividade de doença. A queda da VHS na SAM deve-se a alterações de síntese de proteínas de fase aguda (inclusive fibrinogênio) pelo figado. As alterações das enzimas hepáticas foram vistas nos casos 2 e 3, e a presença de hepatomegalia no caso 3. Na literatura, a presença destes achados é descrita em 33\% a 50\% dos pacientes $^{(1,2,10)}$.

No caso 3, a presença da VHS, que normalizou, dos aumentos de enzimas hepáticas e da pancitopenia foram fatores que sugeriram um quadro compatível com SAM. Apesar das culturas e sorologias não terem sido positivas, não podemos descartar a possibilidade de infecção associada agindo como desencadeante da SAM.

Em relação ao mielograma, não detectamos alterações típicas de SAM, porém a ausência de alterações na medula óssea não invalida o diagnóstico. Acredita-se que a ausência de tais alterações seja dependente da época em que o aspirado de medula é realizado ${ }^{(10)}$. $\mathrm{Na}$ fase inicial, a visualização dos macrófagos ao redor de células hematopoéticas pode estar ausente para surgir algumas semanas depois, devendo o mielograma ser repetido nesse período.

Com relação ao caso 1, cujo mielograma revelou hiperplasia da série eritrocitária e megacariocítica, foram afastadas causas infecciosas que justificassem o quadro clínico e laboratorial. Por outro lado, a normalização da VHS e as alterações das enzimas hepáticas associadas ao quadro clínico nos levaram ao diagnóstico de SAM.

Em um relato de 24 crianças que tiveram 29 episódios de SAM, todas apresentavam alterações nos níveis de coagulação, elevação das enzimas hepáticas, hipertrigliceridemia, diminuição dos níveis de fibrinogênio plasmático e alterações na contagem de plaquetas, compatíveis com o diagnóstico ${ }^{(2)}$. A biópsia evidenciando hemofagocitose foi obtida em 15 aspirados de medula óssea, sendo de consenso que a presença de sinais e sintomas, assim como alterações laboratoriais compatíveis com a síndrome, são suficientes para a confirmação do diagnóstico ${ }^{(2)}$.

Como na literatura, os nossos pacientes beneficiaram-se com o tratamento proposto e aceito pela maioria dos 
autores: pulsoterapia com metilprednisolona, introdução de ciclosporina A e suspensão das drogas imunossupressoras em uso na época do diagnóstico de $\operatorname{SAM}^{(1,2,5,6,10,11)}$. Evolução para óbito foi observada em $8,3 \%$ dos pacientes ${ }^{(2)}$. Na nossa casuística não houve nenhum óbito assim como em outros trabalhos na literatura ${ }^{(5,8,11)}$

\section{REFERÊNCIAS}

1. Cassidy JT, Petty RE: Juvenile Idiopathic Arthritis. Textbook of Pediatric Rheumatology, 4. ${ }^{a}$ ed, Philadelphia, WB Saunders Company, pp. 239-40, 2001.

2. Stéphan JL, Koné-Paut L, Galambrun C, Mouy R, Bader-Meunier B, Prieur AM: Reactive haemophagocytic syndrome in children with inflammatory disorders. A retrospective study of 24 patients. Rheumatology (Oxford) 40: 1285-92, 2001.

3. Risdall RJ, Mc Kenna RW, Nesbit ME, et al: Virus-associated hemophagocytic syndrome: a benign histiocytic proliferation distinct from malignant histiocytosis. Cancer 44: 993-1002, 1979.

4. Hadchouel M, Prieur AM, Griscelli C: Acute Hemorrhagic, hepatic, and neurologic manifestations in juvenile rheumatoid arthritis: possible relationship to drugs or infection. J Pediatr 106: 561-6, 1985.

5. Ceballos LT, Angelin BP, Sánchez JR, Cabrera RD, Narbona FR, Gonzáles JR: Síndrome de activación del macrófago secundario a enfermedad de Still. An Esp Pediatr 51: 194-6, 1999.
Com a descrição destes casos gostaríamos de salientar que o quadro clínico e laboratorial de SAM deve ser levado em consideração, mesmo que o mielograma esteja normal. Salientamos que a SAM é uma complicação rara em crianças com doenças reumáticas, sendo ameaçadora à vida. É importante que seja reconhecida, diferenciando da atividade da AIJ.

6. Imagawa T, Katakura S, Mori M, Aihara Y, Mitsuda T, Yokoda S: A case of macrophage activation syndrome developed with systemic juvenile rheumatoid arthritis. Ryumachi 37: 487-92, 1997.

7. Prahalad S, Bove KE, Dickens D, Lovell DJ, Grom AA: Etanercept in the treatment of macrophage syndrome. J Rheumatol 28: 212024, 2001.

8. Ravelli A, Caria MC, Buratti S, Malattia C, Temporini F, Martini A: Methotrexate as a possible trigger of macrophage activation syndrome in systemic juvenile idiopathic arthritis. J Rheumatol 28: 865-7, 2001

9. Oliveira SKF: Artrite Idiopática Juvenil - Síndrome de Ativação Macrofágica. Reumatologia Pediátrica, 2. ${ }^{a}$ ed, Rio de Janeiro, Revinter, p. 170, 2001.

10. Sawhney S, Woo P, Murray KJ: Macrophage activation syndrome: a potentially fatal complication of rheumatic disorders. Arch Dis Child 85: 421-6, 2001.

11. Cuende E, Vesga JC, Pérez LB, Ardanaz MT, Guinea J: Macrophage activation syndrome as the initial manifestation of systemic onset juvenile idiopathic arthritis. Clin Exp Rheumatol 19: 764-5, 2001. 